\title{
A prevenção da lesão por pressão em unidades de terapia intensiva e a enfermagem neste contexto
}

\author{
Prevention of pressure injury in intensive care units and nursing in this context
}

Prevención de lesiones por presión en unidades de cuidados intensivos y enfermería en este contexto

\author{
Maria Samilly Alves de Araujo ${ }^{*}$, Diogo Jacintho Barbosa²
}

Como citar esse artigo. de Araujo, MSA; Barbosa, DJ. A prevenção da lesão por pressão em unidades de terapia intensiva e a enfermagem neste contexto. Revista Pró-UniverSUS. 2020 Jul./Dez.; 11 (2): 72-79

\begin{abstract}
Resumo
O presente trabalho tem como objeto a produção científica brasileira sobre a prevenção de lesão por pressão em uma unidade de terapia intensiva e a enfermagem neste contexto. A ocorrência de LPP constitui evento adverso em pacentes hospitalizadose, apesar de todos avanços na segurança do paciente, continua acontecendo com elevada incidencia nas UTI. Tendo como objetivo geral: identificar na literatura de que maneira ocorre a atuação da equipe de enfermagem para a prevenção da lesão por pressão nas UTI, e como objetivo específico: descrever as principais intervenções desenvolvidas pelo enfermeiro visando à prevenção da lesão por pressão em uma UTI. Trata-se de uma revisão integrativa, na base de dados da Biblioteca Virtual em Saúde (BVS), sendo os critérios de inclusão artigos completos disponíveis de maneira gratuita, no idioma português e que foram publicados de 2014 a 2019. Foram encontrados 29 artigos que contribuem para o desenvolvimento do estudo e realização do objetivo proposto dentro desta temática. Para melhor compreensão do conteúdo na discussão foram apresentadas as seguintes categorias: as principais intervenções de enfermagem utilizadas na unidade de terapia intensiva para prevenção de lesão por pressão e a capacitação profissional como estratégia para redução de riscos relacionados a lesão por pressão e os desafios encontrados. Conclui-se que a educação permanente é fator primordial para capacitação profissional. É necessário que as instituições priorizem a elaboração e a implementação de protocolos de prevenção, bem como, trabalhar com um número ideal de enfermeiros, a fim de evitar sobrecarga de trabalho, melhorando assim a qualidade assistencial.
\end{abstract}

Palavras-chave: Enfermagem, Lesão por Pressão, Unidade Intensiva.

\begin{abstract}
The present work has as its object the Brazilian scientific production on the prevention of pressure injuries in an intensive care unit and nursing in this context. The occurrence of LPP is an adverse event in hospitalized patients and, despite all advances in patient safety, it continues to happen with a high incidence in ICUs. Having as general objective: to identify in the literature how the nursing team works to prevent pressure injuries in the ICU, and as a specific objective: to describe the main interventions developed by nurses aiming at the prevention of pressure injuries in an ICU . It is an integrative review, in the database of the Virtual Health Library (VHL), with the inclusion criteria being full articles available free of charge, in the Portuguese language and that were published from 2014 to 2019. 29 articles were found that contribute to the development of the study and achievement of the proposed objective within this theme. For a better understanding of the content in the discussion, the following categories were presented: the main nursing interventions used in the intensive care unit to prevent pressure injuries and professional training as a strategy to reduce risks related to pressure injuries and the challenges encountered. It is concluded that permanent education is a key factor for professional training. It is necessary for institutions to prioritize the development and implementation of prevention protocols, as well as working with an ideal number of nurses, in order to avoid work overload, thus improving the quality of care.
\end{abstract}

Keywords: Nursing, Pressure Injury, Intensive Unit. 


\section{Resumen}

El presente trabajo tiene como objeto la producción científica brasileña sobre la prevención de lesiones por presión en una unidad de cuidados intensivos y la enfermería en este contexto. La ocurrencia de LPP es un evento adverso en pacientes hospitalizados y, a pesar de todos los avances en seguridad del paciente, continúa ocurriendo con una alta incidencia en UCI. Teniendo como objetivo general: identificar en la literatura cómo trabaja el equipo de enfermería para la prevención de lesiones por presión en la UCI, y como objetivo específico: describir las principales intervenciones desarrolladas por enfermeras con el objetivo de prevenir las lesiones por presión en una UCI . Se trata de una revisión integradora, en la base de datos de la Biblioteca Virtual en Salud (BVS), siendo los criterios de inclusión artículos completos disponibles de forma gratuita, en idioma portugués y que fueron publicados entre 2014 y 2019.Se encontraron 29 artículos que Contribuir al desarrollo del estudio y logro del objetivo propuesto dentro de esta temática. Para una mejor comprensión del contenido de la discusión, se presentaron las siguientes categorías: las principales intervenciones de enfermería utilizadas en la unidad de cuidados intensivos para prevenir las lesiones por presión y la formación profesional como estrategia para reducir los riesgos relacionados con las lesiones por presión y los desafíos encontrados. Se concluye que la educación permanente es un factor clave para la formación profesional. Es necesario que las instituciones prioricen el desarrollo e implementación de protocolos de prevención, así como trabajar con un número ideal de enfermeras, a fin de evitar la sobrecarga laboral, mejorando así la calidad de la atención.

Palabras clave: Enfermería, Lesión por presión, Unidad intensiva.

\section{Introdução}

\section{O National Pressure Ulcer Advisory Panel} (NPUAP), em 2007, atualizou a definição de úlcera por pressão e o seu sistema de classificação e, em 2016, a sua terminologia de úlcera por pressão para Lesão por Pressão (LPP), a partir de então, a LPP passou a ser definida como uma lesão localizada na pele e/ou no tecido ou estrutura subjacente, geralmente sobre uma proeminência óssea, resultante de pressão intensa e/ou prolongada ou de pressão em combinação com cisalhamento. Desta forma, os pacientes de uma unidade de terapia intensiva (UTI), tornam-se vulneráveis a desenvolvê-las, devido às restrições impostas, associadas às condições clínicas graves e terapias de maior complexidade ${ }^{1}$.

Visto a necessidade de um atendimento integral e seguro, foi instituído pelo Ministério da Saúde o Programa Nacional de Segurança do Paciente (PNSP), Portaria 529/2013, em $1^{\circ}$ de abril de 2013, que a prevenção de lesões por pressão compõem os seis eixos para prevenção de dano, contribuindo para a qualificação do cuidado em saúde, em todos os estabelecimentos de saúde do território Nacional brasileiro, sendo o enfermeiro essencial para esse processo de avaliação, prevenção e redução de riscos de eventos adversos ${ }^{2}$.

Apesar de todo avanço científico e tecnológico na área da saúde, a incidência de lesões por pressão permanece sendo um dos principais problemas de saúde, devido aos longos períodos de internação, podendo agravar o quadro clínico do paciente, levando a complicações no estado de saúde do paciente, desta forma, a intervenção precoce torna-se necessária, tendo como objetivo o cuidado focado na prevenção e promoção da segurança do paciente em tratamento ${ }^{3}$.

O desenvolvimento da LPP causa danos significativos ao paciente, dificultando o processo de recuperação funcional. Podendo gerar dor e levar ao desenvolvimento de graves infecções, assim como tem sido associado a internações prolongadas, sepse e mortalidade ${ }^{4}$.

A prevenção de lesão por pressão nos pacientes hospitalizados não é tão complicada, a medida mais simples e comum é a mudança periódica da posição do paciente. É muito importante que todos os envolvidos no cuidado tenham conhecimento de como tratar de maneira adequada as lesões por pressão, a fim de evitálas 5 .

A partir desta premissa surgiu como pergunta de pesquisa: como a enfermagem pode influenciar para o prognóstico, desenvolvimento e prevenção das LPP?

A fim de responder esta questão foram traçados como objetivo geral deste estudo: Identificar na literatura de que maneira ocorre a atuação da equipe de enfermagem para a prevenção da lesão por pressão nas UTI, e como objetivo específico: descrever as principais intervenções desenvolvidas pelo enfermeiro visando à prevenção da lesão por pressão em uma unidade de Terapia Intensiva.

\section{Metodologia}

Trata-se de um estudo para revisão bibliográfica. Para a realização desta análise, foi utilizado como base a Biblioteca Virtual em Saúde, sendo utilizadas as seguintes palavras chave: enfermagem, lesão por pressão e terapia intensiva. Os critérios de inclusão baseiam-se em artigos completos disponíveis de maneira gratuita, no idioma português e que foram publicados de 2014 a 2019. A busca dos artigos deu-se entre os meses de fevereiro a abril de 2020, onde obtive o retorno de 242 artigos, após aplicação dos filtros e leitura prévia resultou em uma amostra final de 29 artigos. Para melhor entendimento do conteúdo encontrado fiz uma leitura analítica e interpretativa que possibilitou nos resultados a construção de três tabelas trazendo a amostra final do estudo, a distribuição dos artigos por ano de publicação 
e os tipos de pesquisas em cada artigo e na discussão me permitiu categorizar dois assuntos que mais foram abordados nos artigos selecionados.

A abordagem qualitativa facilita descrever a complexidade e as hipóteses, bem como analisar a interação entre variáveis, compreender e classificar determinados processos sociais, oferecer contribuições no processo de mudanças, criação ou formação de opiniões de determinados grupos e interpretação das particularidades dos comportamentos ou atitudes dos indivíduos ${ }^{6}$.

Ao ensinar sua metodologia Lakatos (2003), vem demonstrando de forma lúcida e clara métodos e conceitos para melhor formulação de uma pesquisa, tanto no aspecto prático e visual, como também na forma de observação, descrição, análise e conclusão de eventos, através da conceituação e exemplificação de métodos, fatos, leis e hipóteses. Sua metodologia traz grande enfoque para a leitura que deve ser proveitosa e trazer resultados satisfatórios, pois a leitura representa fator decisivo, acrescenta novos conhecimentos, abrindo novos horizontes para a mente, coordenando o pensamento, enriquecendo o vocabulário e facilitando o entendimento do conteúdo da obra. A leitura continuada e constante é essencial para assimilação, retenção, crítica, comparação, verificação e integração do conhecimento. Assim, o método é o conjunto das atividades sistemáticas e racionais que, com maior segurança e economia, permite alcançar o objetivo - conhecimentos válidos e verdadeiros -, traçando o caminho a ser seguido, detectando erros e auxiliando as decisões do cientista ${ }^{6}$.

\section{Resultados e Discussão}

A busca na base de dados retornou 242 artigos, após a aplicação dos filtros, encontramos 41 artigos, estes foram selecionados para leitura o que resultou em uma amostra final de 29 artigos. A Tabela 1 demonstra os artigos que compõem a amostra final deste estudo e nos proporcionou uma avaliação geral dos artigos.

Com base no ano de publicação de artigos observa-se que o ano com maior número de publicações foi em 2018, seguido pelos demais anos conforme demonstrado na Tabela 2.

$\mathrm{Na}$ tabela 3 podemos verificar a distribuição dos artigos pelo tipo de pesquisa, na qual foi possível observar que a pesquisa qualitativa nos seus dois moldes, descritivo e transversal foi o tipo de pesquisa que apresentou maior número de estudos (20), seguidos pela pesquisa quantitativa.

No que concerne à forma de publicação, a maioria dos artigos foram publicados em revistas online, todas direcionadas à saúde, sendo 26 artigos publicados em revistas de enfermagem. Todas as pesquisas foram feitas em diversas regiões do Brasil.

Neste estudo, foi possível observar, a partir da leitura de todos os artigos, que a maior parte dos profissionais intensivistas demonstram compreensão dos cuidados relacionados à prática profissional, que o planejamento de risco é imprescindível, da importância da intervenção para prevenção da lesão por pressão e da capacitação profissional como estratégia para reduzir riscos. Dessa forma, para melhor compreensão do conteúdo na discussão serão apresentadas as seguintes categorias:

\section{As principais intervenções de enfermagem utilizadas na unidade de terapia intensiva para prevenção de lesão por pressão}

A NANDA internacional define risco de lesão por pressão como um estado de vulnerabilidade de dano celular na pele e no tecido subjacente, resultante da compressão dos tecidos moles, geralmente sobre uma proeminência óssea, durante um período de tempo capaz de ocasionar isquemia local e, consequentemente, necrose. Conhecer os fatores de risco favorece para interferência diagnóstica e otimiza à tomada de decisão clínica dos enfermeiros, além de orientar as intervenções de enfermagem preventiva para diminuir a incidência desse agravo e alcançar resultados positivos em saúde ${ }^{7}$.

Com base nos artigos é possível afirmar que pesquisas recentes evidenciam altosíndices de ocorrência das lesões de pele como a LPP em pacientes na terapia intensiva. $\mathrm{O}$ enfermeiro é o profissional que está à frente no cuidado ao paciente e deve ter competência para o gerenciamento de implementação de estratégias para a prevenção de danos aos pacientes, as quais devem visar à eliminação dos riscos e à minimização de processos prejudiciais ${ }^{8,9}$.

De acordo com os artigos estudados na revisão integrativa, a Escala de Braden é um instrumento que prever por antecipação diagnóstico de risco para lesão por pressão, no qual avalia itens como percepção sensorial, nutrição, umidade, atividade, fricção e cisalhamento e mobilidade, sendo apontada como estratégia importante na prevenção de lesões por pressão em unidades críticas $^{10,11}$

Os enfermeiros reconhecem que a avaliação do paciente, identificação dos fatores de riscos e implementação de um plano de prevenção de LPP baseado nos riscos apresentados pelos pacientes, e o uso de colchão piramidal de espuma, são importantes medidas preventivas a serem aplicadas e são citadas nos diversos artigos estudados ${ }^{3,4}$.

O reposicionamento é descrito como a forma de prevenção de LP mais conhecida e utilizada pela enfermagem. Todavia, no artigo "Intervenções de enfermeiros na prevenção de lesão por pressão em uma 
Tabela 1. Apresentação dos artigos que compõem a amostra final do estudo.

\begin{tabular}{|c|c|c|c|c|c|}
\hline Autor & Título & Revista & Ano & Tipo de Pesquisa & País \\
\hline $\begin{array}{l}\text { Barreto, Rena- } \\
\text { ta Michelle Dos } \\
\text { Santos }\end{array}$ & $\begin{array}{l}\text { Perfil de pessoas com lesão por pressão in- } \\
\text { ternados na unidade de terapia intensiva }\end{array}$ & $\begin{array}{l}\text { Mundo saúde } \\
\text { (Impr.) }\end{array}$ & 2019 & $\begin{array}{l}\text { Retrospectiva, descritiva } \\
\text { estudo com abordagem } \\
\text { quantitativa }\end{array}$ & Brasil \\
\hline $\begin{array}{l}\text { Manganelli, Ri- } \\
\text { gielli Ribeiro }\end{array}$ & $\begin{array}{l}\text { Intervenções de enfermeiros na prevenção } \\
\text { de lesão por pressão em uma unidade de } \\
\text { terapia intensiva }\end{array}$ & $\begin{array}{l}\text { Rev. enferm. } \\
\text { UFSM }\end{array}$ & 2019 & Transversal Descritiva & Brasil \\
\hline $\begin{array}{l}\text { Farias, Ana Dark } \\
\text { Aires de }\end{array}$ & $\begin{array}{c}\text { Ocorrência de lesões por pressão em uni- } \\
\text { dade de terapia intensiva de um hospital } \\
\text { universitário }\end{array}$ & $\begin{array}{l}\text { Nursing (Säo Pau- } \\
\text { lo) }\end{array}$ & 2019 & $\begin{array}{c}\text { Descritiva, longitudinal e } \\
\text { qualitativa }\end{array}$ & Brasil \\
\hline Otto, Carolina & $\begin{array}{l}\text { Fatores de risco para o desenvolvimento de } \\
\text { lesão por pressão em pacientes críticos }\end{array}$ & $\begin{array}{l}\text { Enferm. foco } \\
\text { (Brasília) }\end{array}$ & 2019 & Descritivo quantitativo & Brasil \\
\hline $\begin{array}{l}\text { Caldini, Luana } \\
\text { Nunes }\end{array}$ & $\begin{array}{c}\text { Avaliação de tecnologia educativa sobre le- } \\
\text { são por pressão baseada em indicadores de } \\
\text { qualidade assistenciais }\end{array}$ & $\begin{array}{l}\text { Rev Rene } \\
\text { (Online) }\end{array}$ & 2018 & Experimental & Brasil \\
\hline $\begin{array}{l}\text { Oliveira Sanches, } \\
\text { Bruna }\end{array}$ & $\begin{array}{c}\text { Adesão da enfermagem ao protocolo de } \\
\text { lesão por pressão em unidade de terapia } \\
\text { intensiva }\end{array}$ & $\begin{array}{l}\text { Arch. Health Sci. } \\
\qquad \text { (Online) }\end{array}$ & 2018 & Quantitativo & Brasil \\
\hline $\begin{array}{l}\text { Silva, Makcine } \\
\text { Timm da }\end{array}$ & $\begin{array}{c}\text { Prevenção de complicações evitáveis em } \\
\text { uma unidade de terapia intensiva: uma re- } \\
\text { visão integrativa }\end{array}$ & $\begin{array}{l}\text { Saúde e pesqui. } \\
\text { (Impr.) }\end{array}$ & 2018 & Revisão Integrativa & Brasil \\
\hline $\begin{array}{l}\text { Barbosa, Taís Pa- } \\
\text { gliuco }\end{array}$ & $\begin{array}{l}\text { Associação entre sedação e eventos adver- } \\
\text { sos em pacientes de terapia intensiva }\end{array}$ & $\begin{array}{c}\text { Acta Paul. } \\
\text { Enferm. (Online) }\end{array}$ & 2018 & $\begin{array}{c}\text { Retrospectivo e Quantita- } \\
\text { tivo }\end{array}$ & Brasil \\
\hline $\begin{array}{l}\text { Mendonça, Paula } \\
\text { Knoch }\end{array}$ & $\begin{array}{l}\text { Ocorrência e fatores de risco para lesões } \\
\text { por pressão em centros de terapia intensiva }\end{array}$ & $\begin{array}{l}\text { Rev. enferm. } \\
\text { UFPE (online) }\end{array}$ & 2018 & Quantitativo, transversal & Brasil \\
\hline $\begin{array}{l}\text { Souza, Ragive } \\
\text { Ferreira de }\end{array}$ & $\begin{array}{c}\text { Eventos adversos na Unidade de Terapia } \\
\text { Intensiva }\end{array}$ & $\begin{array}{l}\text { Rev. enferm. } \\
\text { UFPE (online) }\end{array}$ & 2018 & $\begin{array}{l}\text { Retrospectivo, quantitati- } \\
\text { vo, descritivo }\end{array}$ & Brasil \\
\hline $\begin{array}{l}\text { Holanda, Odair } \\
\text { Queiroz de }\end{array}$ & $\begin{array}{c}\text { Efetividade do protocolo para prevenção de } \\
\text { lesões por pressão implantado em Unidade } \\
\text { de Terapia Intensiva }\end{array}$ & $\begin{array}{l}\text { Espaç. saúde } \\
\text { (Online) }\end{array}$ & 2018 & $\begin{array}{l}\text { Quantitativo descritivo, } \\
\text { analítico e documental }\end{array}$ & Brasil \\
\hline $\begin{array}{l}\text { Zimmermann, } \\
\text { Guilherme dos } \\
\text { Santos }\end{array}$ & $\begin{array}{l}\text { Predição de risco de lesão por pressão em } \\
\text { pacientes de unidade de terapia intensiva: } \\
\text { revisão integrativa }\end{array}$ & $\begin{array}{c}\text { Texto \& contexto } \\
\text { enferm }\end{array}$ & 2018 & Revisão Integrativa & Brasil \\
\hline $\begin{array}{l}\text { Medeiros, Luan } \\
\text { Nogueira Bezer- } \\
\text { ra de }\end{array}$ & $\begin{array}{l}\text { Prevalência de úlceras por pressão em uni- } \\
\text { dades de terapia intensiva }\end{array}$ & $\begin{array}{l}\text { Rev. enferm. } \\
\text { UFPE (online) }\end{array}$ & 2017 & Transversal, quantitativa & Brasil \\
\hline $\begin{array}{l}\text { Silva, Dinara Ra- } \\
\text { quel Araújo }\end{array}$ & $\begin{array}{l}\text { Curativos de lesões por pressão em pacien- } \\
\text { tes críticos: análise de custos }\end{array}$ & $\begin{array}{l}\text { Rev Esc Enferm } \\
\text { USP }\end{array}$ & 2017 & Observacional descritivo & Brasil \\
\hline $\begin{array}{l}\text { Ortega, Daniela } \\
\text { Benevides }\end{array}$ & $\begin{array}{l}\text { Análise de eventos adversos em pacientes } \\
\text { internados em unidade de terapia intensiva }\end{array}$ & $\begin{array}{l}\text { Acta Paul. Enferm. } \\
\text { (Online) }\end{array}$ & 2017 & $\begin{array}{c}\text { Transversal, prospectivo, } \\
\text { com abordagem quantita- } \\
\text { tiva }\end{array}$ & Brasil \\
\hline $\begin{array}{l}\text { Aredes, Natália } \\
\text { Del Angelo }\end{array}$ & $\begin{array}{c}\text { Cuidados com a pele do recém-nascido } \\
\text { prematuro: revisão integrativa }\end{array}$ & $\begin{array}{l}\text { Rev. eletrônica en- } \\
\text { ferm }\end{array}$ & 2017 & Revisão Integrativa & Brasil \\
\hline $\begin{array}{l}\text { Vasconcelos, Jo- } \\
\text { silene de Melo } \\
\text { Buriti }\end{array}$ & $\begin{array}{c}\text { Ações de enfermagem antes e após um pro- } \\
\text { tocolo de prevenção de lesões por pressão } \\
\text { em terapia intensiva }\end{array}$ & $\begin{array}{l}\text { Esc. Anna Nery } \\
\text { Rev. Enferm }\end{array}$ & 2017 & $\begin{array}{l}\text { Observacional, prospec- } \\
\text { tivo, comparativo com } \\
\text { abordagem quantitativa. }\end{array}$ & Brasil \\
\hline $\begin{array}{l}\text { Leitão, Ilse Ma- } \\
\text { ria Tigre de Ar- } \\
\text { ruda }\end{array}$ & $\begin{array}{l}\text { Absenteísmo, rotatividade e indicadores de } \\
\text { qualidade do cuidado em enfermagem: es- } \\
\text { tudo transversal }\end{array}$ & $\begin{array}{l}\text { Online braz. j. nurs. } \\
\text { (Online) }\end{array}$ & 2017 & Transversal, quantitativo & Brasil \\
\hline $\begin{array}{l}\text { Toffoletto, Maria } \\
\text { Cecília }\end{array}$ & $\begin{array}{l}\text { Fatores relacionados à ocorrência de even- } \\
\text { tos adversos em pacientes idosos críticos. }\end{array}$ & Rev Bras Enferm & 2016 & $\begin{array}{l}\text { Estudo de coorte retros- } \\
\text { pectivo }\end{array}$ & Brasil \\
\hline $\begin{array}{l}\text { Silva, Camila } \\
\text { Polo Camargo da }\end{array}$ & $\begin{array}{l}\text { Construção do Aplicativo para o indicador } \\
\text { de úlcera por pressão }\end{array}$ & $\begin{array}{l}\text { Informática Médica } \\
\text { / Serviços de Saúde } \\
\text { / TECNOLOGIA }\end{array}$ & 2016 & Descritivo & Brasil \\
\hline $\begin{array}{l}\text { Baron, Mirian } \\
\text { Viviane }\end{array}$ & $\begin{array}{l}\text { Estudo experimental com equipes de En- } \\
\text { fermagem acerca do conhecimento sobre } \\
\text { úlceras por pressão }\end{array}$ & $\begin{array}{l}\text { Rev Lat Am Enfer- } \\
\text { magem }\end{array}$ & 2016 & $\begin{array}{l}\text { Quantitativo com delinea- } \\
\text { mento experimental }\end{array}$ & Brasil \\
\hline
\end{tabular}


Tabela 1 (cont.). Apresentação dos artigos que compõem a amostra final do estudo.

\begin{tabular}{|c|c|c|c|c|c|}
\hline Autor & Título & Revista & Ano & Tipo de Pesquisa & País \\
\hline $\begin{array}{l}\text { Oliveira, Andrea } \\
\text { Carvalho de }\end{array}$ & $\begin{array}{l}\text { Carga de trabalho de enfermagem e ocor- } \\
\text { rência de eventos adversos na terapia inten- } \\
\text { siva: revisão sistemática }\end{array}$ & $\begin{array}{l}\text { Rev Esc Enferm } \\
\text { USP }\end{array}$ & 2016 & $\begin{array}{l}\text { Revisão sistemática da li- } \\
\text { teratura }\end{array}$ & Brasil \\
\hline $\begin{array}{l}\text { Baquehais, Adna } \\
\text { Ribeiro }\end{array}$ & $\begin{array}{l}\text { Conhecimento dos enfermeiros acerca da } \\
\text { prevenção de lesões por pressão em unida- } \\
\text { de de terapia intensiva }\end{array}$ & $\begin{array}{l}\text { Rev. enferm. } \\
\text { UFPI }\end{array}$ & 2016 & Transversal, descritivo & Brasil \\
\hline Olkoski, Elaine & $\begin{array}{l}\text { Aplicação de medidas de prevenção para } \\
\text { úlceras por pressão pela equipe de enfer- } \\
\text { magem antes e após uma campanha edu- } \\
\text { cativa }\end{array}$ & $\begin{array}{l}\text { Esc. Anna Nery } \\
\text { Rev. Enferm }\end{array}$ & 2016 & $\begin{array}{l}\text { Exploratório-descritiva } \\
\text { com abordagem quantita- } \\
\text { tiva }\end{array}$ & Brasil \\
\hline $\begin{array}{l}\text { Busanello, Jose- } \\
\text { fine }\end{array}$ & $\begin{array}{l}\text { Cuidados de enfermagem ao paciente adul- } \\
\text { to: prevenção de lesões cutaneomucosas e } \\
\text { segurança do paciente }\end{array}$ & $\begin{array}{l}\text { Rev. enferm. } \\
\text { UFSM }\end{array}$ & 2015 & $\begin{array}{l}\text { Descritivo e exploratório, } \\
\text { de abordagem qualitativa }\end{array}$ & Brasil \\
\hline $\begin{array}{l}\text { Lemos, Débora } \\
\text { de Sousa }\end{array}$ & $\begin{array}{l}\text { Conhecimentos dos profissionais de enfer- } \\
\text { magem relacionados às úlceras por pressão }\end{array}$ & $\begin{array}{l}\text { Rev. enferm. } \\
\text { UFSM }\end{array}$ & 2014 & Quantitativo & Brasil \\
\hline $\begin{array}{l}\text { Dantas, Anna Lí- } \\
\text { via de Medeiros }\end{array}$ & $\begin{array}{l}\text { Prática do enfermeiro intensivista no trata- } \\
\text { mento de úlceras por pressão }\end{array}$ & $\begin{array}{l}\text { Rev. pesqui. cuid. } \\
\text { fundam. (Online) }\end{array}$ & 2014 & Descritivo & Brasil \\
\hline $\begin{array}{l}\text { Alves, Ana Gle- } \\
\text { cia Pimentel }\end{array}$ & $\begin{array}{l}\text { Avaliação do risco para úlcera por pressão } \\
\text { em unidades de terapia intensiva: uma revi- } \\
\text { são integrativa }\end{array}$ & $\begin{array}{l}\text { Rev. pesqui. cuid. } \\
\text { fundam. (Online) }\end{array}$ & 2014 & Revisão Integrativa & Brasil \\
\hline $\begin{array}{l}\text { Albuquerque, } \\
\text { Adriana Monte- } \\
\text { negro }\end{array}$ & $\begin{array}{l}\text { Avaliação e prevenção da úlcera por pres- } \\
\text { são pelos enfermeiros de terapia intensiva: } \\
\text { conhecimento e prática }\end{array}$ & $\begin{array}{l}\text { Rev. enferm. } \\
\text { UFPE (online) }\end{array}$ & 2014 & $\begin{array}{l}\text { Exploratório, com aborda- } \\
\text { gem quantitativa }\end{array}$ & Brasil \\
\hline
\end{tabular}

Fonte: Pesquisa dos Autores.

Tabela 2. Distribuição dos artigos por ano de publicação.

\begin{tabular}{ccc}
\hline Ano de Publicação & $\mathbf{N}^{\mathbf{0}}$ & $\mathbf{\%}$ \\
\hline 2019 & 4 & $14 \%$ \\
\hline 2018 & 8 & $27 \%$ \\
\hline 2017 & 6 & $22 \%$ \\
\hline 2016 & 6 & $22 \%$ \\
\hline 2015 & 1 & $3 \%$ \\
\hline 2014 & 4 & $14 \%$ \\
\hline Total & 29 & $100 \%$ \\
\hline
\end{tabular}

Fonte: Pesquisa dos Autores
Tabela 3. Distribuição dos artigos pela metodologia de estudos.

\begin{tabular}{cll}
\hline Tipo de pesquisa & $\mathbf{N}^{\mathbf{0}}$ & $\mathbf{\%}$ \\
\hline Quantitativa & 15 & 52 \\
\hline Qualitativa - Descritiva & 13 & 45 \\
\hline Qualitativa - Transversal & 7 & 24 \\
\hline Revisão integrativa & 5 & 17 \\
\hline Prospectiva & 4 & 14 \\
\hline Estudo de Coorte & 3 & 10 \\
\hline Explanatória & 3 & 10 \\
\hline Documental & 2 & 7 \\
\hline Qualitativo & 2 & 7 \\
\hline Observacional & 2 & 7 \\
\hline Longitudinal & 2 & 7 \\
\hline Retrospectivo & 2 & 7 \\
\hline Comparativo & 1 & 3 \\
\hline Analítico & 1 & 105 \\
\hline
\end{tabular}

Fonte: Pesquisa dos Autores. 
unidade de terapia intensiva", a literatura contempla um estudo realizado com pacientes em uma UTI Adulto de um hospital de médio porte, da região da fronteira oeste do Rio Grande do Sul (RS), nesta pesquisa ficou evidente a omissão desse cuidado, fato que vai ao encontro de um estudo que identificou que o reposicionamento do paciente a cada duas horas, ou conforme necessário, foi omitido por $50(31,1 \%)$ dos enfermeiros. Enfatizase que uma nutrição inadequada pode influenciar no desenvolvimento de LPP, pois um organismo nessas condições apresenta alterações no transporte de oxigênio e no metabolismo ${ }^{8}$.

A incidência e a prevalência de lesões por pressão nos cenários assistenciais são variáveis ${ }^{11}$, no qual identifica-se, nos Estados Unidos, em pacientes críticos, prevalência de $8,8 \%$ a $9,3 \%{ }^{12}$. Em contrapartida no Brasil, essas taxas em idosos internados correspondem a $18,8 \%$ e, em pacientes de unidades de terapia intensiva, correspondem de $17,7 \%$ a $35,2 \%{ }^{11}$.

No artigo sobre "Predição de risco de lesão por pressão em pacientes de unidade de terapia intensiva: revisão integrativa" é possível identificar que Norton, Gosnell, Waterlow e Braden, são as escalas para avaliação de risco para LPP mais conhecidas e aplicadas são, sendo a última a mais utilizada em adultos e estudada no Brasil e destaca como um bom instrumento para predição de risco. A utilização de uma escala adequada pode trazer benefícios como a diminuição de incidências de lesões por pressão, assim como a mudança de decúbito a cada duas horas, a proteção de proeminências ósseas, redução da exposição da pele a umidade, dentre outros. Todavia, se tratando de paciente críticos, devese levar em consideração as peculiaridades de sua condição clínica durante a avaliação de risco para LPP. Nota-se que há necessidade de maiores estudos para intervenções de risco para esses pacientes. Em alguns outros artigos reforçam a importância de implementar medidas preventivas com embasamento científico para assistência de enfermagem ao paciente crítico $^{13}$.

Considera-se que a LPP pode ser evitada, a partir da capacitação da equipe quanto à identificação dos fatores de riscos. A partir desta informação, surge nossa segunda categoria:

\section{A capacitação profissional como estratégia para redução de riscos relacionados a lesão por pressão e os desafios encontrados}

A investigação e análise da demanda de treinamento, configura-se etapa preliminar e principal da organização de práticas didáticas realizadas pela educação permanente, pois interliga continuamente os cenários sociais, assistenciais e educacionais, além de promover aos profissionais de saúde a disposição e a capacidade necessárias ao discernimento sobre as dificuldades e necessidades do trabalho em saúde e qualificação profissional ${ }^{14}$.

Programas de capacitação e treinamento são essenciais à análise e à implementação de indicadores assistenciais, para que haja acompanhamento da qualidade da assistência e da necessidade de reorientação, garantindo assim promoção da segurança do paciente e melhoria da prática profissional na ocorrência e gravidade de eventos adversos ${ }^{11}$.

Essa situação-problema, por meio de recursos de ensino, permite retratar fatores no cotidiano do enfermeiro e da assistência de enfermagem, como por exemplo a ocorrência de lesão por pressão, caracterizada como um indicador negativo de qualidade do cuidado, e avaliada internacionalmente como evento adverso, representando assim um importante desafio para o cuidado em saúde, por contribuir com o aumento da morbidade, da mortalidade, tempo, custos do tratamento de saúde e afetar elevado número de pessoas ${ }^{15}$.

Esses procedimentos são demandas diárias e inerentes à profissão, onde os enfermeiros ao prestarem assistência, deparam-se com uma realidade onde a população requer as medidas procedimentais de cuidado, que envolvem antes de tudo, a identificação do problema e posteriormente a resolução do mesmo. Sendo assim, complementa a autora que "a aprendizagem relacionada ao cuidado nas necessidades do cliente se faz necessária"16,17.

Rolim, retrata em sua pesquisa a relevância do capital material de prevenção de lesão por pressão, onde somente viabiliza a carência do trabalho empreendido, pois seu propósito prático-assistencial é indispensável ao desenvolvimento das ações e cuidados de enfermagem. Para tal, necessita-se de qualificação constante e organizada, baseada em evidências científicas inovadoras e contemporâneas para o emprego adequado desses dispositivos, reduzindo assim, a incidência de danos ao paciente ${ }^{18,19}$.

De acordo com a literatura é preciso identificar e trabalhar as limitações encontradas na prática, sendo que, as principais dificuldades apontadas foram relacionadas à falta de registros de enfermagem e carência de recursos humanos, além da falta de capacitações e educação permanente acerca da prevenção de LPP e limitações de recursos materiais ${ }^{20,21,22,23}$. De acordo com alguns achados, é possível associar a omissão de cuidados a fatores relacionados a recursos humanos, devido ao o número insuficiente de profissionais da enfermagem relacionado com a sobrecarga de trabalho ${ }^{24}$.

Podemos destacar que a alocação inapropriada da equipe de enfermagem encontra-se correlacionada à elevada carga trabalhista, já que alguns dos princípios precipitadores das lesões por pressão envolvem a postergação das intervenções preventivas, em decorrência da elevada demanda do processo de trabalho 
em saúde ${ }^{24}$.

Adespeito do progressivo receio dos trabalhadores em saúde em propiciar cuidado qualificado e seguro, fundamentado por condutas científicas e estabelecimento de procedimentos voltados à prevenção de feridas, entre elas, as lesões por pressão, sendo necessário ainda, considerar os inúmeros obstáculos encontrados nas organizações de saúde, como por exemplo, a insuficiência de pessoal ${ }^{25}$.

Em alguns artigos, é citado como desafio o aumento do número de idosos no Brasil. O artigo revela que entre 2000 e 2050, a proporção de pessoas maiores de 60 anos se duplicará, passando de 11\% a $22 \%{ }^{26}$. A literatura informa que as lesões por pressão se tornam mais frequente em idosos, assim como os EA com maior permanência e tempo de ventilação invasiva na UTI e os acidentes o que leva a uma sobrecarga de trabalho maior quando as alocações de profissionais da enfermagem não estão adequadas. A mortalidade dos idosos em UTI é abordada em diversos estudos. Neste estudo, 38,3\% dos idosos que sofreram EA moderados e graves foram a óbito ${ }^{27}$.

\section{Considerações Finais}

O estudo buscou evidenciar que a enfermagem ao longo dos anos tem produzido conhecimentos que procuram aprimorar e qualificar a prática profissional, para tanto, a preocupação em escrever sobre a prevenção de lesão por pressão, já que as LPP constituem um grave problema de saúde pública, principalmente em razão do impacto que causam aos doentes, sua família e a sociedade.

Nas unidades de terapia intensiva, esse evento é um problema constante, pois sua evolução é rápida, trazendo risco de complicações ao paciente internado, prolongando seu tempo de tratamento e reabilitação.

Levando em consideração os estudos analisados, pode-se identificar que a enfermagem, de forma direta e indireta, tem importância fundamental no cuidado aos pacientes hospitalizados, principalmente quando o assunto está relacionado à prevenção de lesão por pressão. Podemos constatar que a educação permanente é fator primordial para capacitação profissional, melhorando a abordagem de cuidados com evidências científica, todavia, é necessário que as instituições priorizem a elaboração e a implementação de protocolos de prevenção, bem como, trabalhar com um número ideal de enfermeiros e técnicos de enfermagem, a fim de evitar sobrecarga de trabalho, melhorando assim a qualidade assistencial.

\section{Referências}

1. National Pressure Ulcer Advisory Panel (NPUAP). Classificação das lesões por pressão. Adaptada culturamente para o Brasil por Maria Helena Larcher Caliri, Vera lucia Conceição de Gouveia Santos, Maria Helena Santana Madelbaum e Idevania Geraldina Costa. Publicação oficial da Associação Brasileira de Estomaterapia - SOBEST e da Associação Brasileira de enfermagem em Dermatologia - SOBENDE, 2016. Disponível em: < http://www.sobest.org.br/textod/35>. Acesso em: 19 mar. 2020.

2. Brasil. Ministério da Saúde. Gabinete do Ministro. Portaria $\mathrm{n}^{0} 529$, de $1^{\circ}$ de abril de 2013. Brasislia. Disponivel em: < http://bvsms.saude.gov. br/bvs/saudelegis/gm/2013/prt0529_01_04_2013.html > Acesso em: $30 \mathrm{mar}$ 2020.

3. Brasil. Agência Nacional de Vigilância Sanitária - ANVISA NOTA TÉCNICA GVIMS/GGTES No 03/2017 Práticas seguras para prevenção de Lesão por Pressão em serviços de. Saúde. Disponível em: <http://portal. anvisa.gov.br/documents/33852/271855/Nota + T\%C3\%A9cnica+GVIMSG GTES +n\%C2\%BA+03-2017/54ec39f6-84e0-4cdb-a241-31491ac6e03e > Acesso em: 04 Abr 2020.

4. Olkoski, Elaine; Assis, Gisela Maria. Aplicação de medidas de prevenção para úlceras por pressão pela equipe de enfermagem antes e após uma campanha educativa. Esc. Anna Nery, Rio de Janeiro, v. 20, n. 2, p. $363-$ 369, June 2016. Available from $<\mathrm{http} / /$ www.scielo.br/scielo.php?script $=$ sci arttext\&pid=S1414-81452016000200363\&lng $=$ en\&nrm $=$ iso $>$. access on $2 \overline{9}$ June. 2020. https://doi.org/10.5935/1414-8145.20160050.

5. Lima, Paula Rodrigues et al. Ocorrência de lesão por pressão em pacientes hospitalizados: uma revisão integrativa. REVISTA UNINGÁ REVIEW, [S.1.], v. 32, n. 1, p. 53 - 67, out. 2017. ISSN 2178-2571. Disponível em: $\quad<$ http://revista.uninga.br/index.php/uningareviews/article/view/39>. Acesso em: 26 abr. 2020

6. Lakatos, Eva Maria; Marconi, Marina de Andrade. Fundamentos de Metodologia Cientifica [Internet]. 5. ed. - São Paulo: Atlas 2003. Disponivel em: <https://docente.ifrn.edu.br/olivianeta/disciplinas/copy_of_historia-i/ historia-ii/china-e-india $>$. Acesso em: 16 Mar. 2020

7. Nanda Internacional. Diagnósticos de enfermagem da NANDA-I (2018- 2020). Tradução de Regina Machado Garcez. 11. ed. Porto Alegre (RS): Artmed, 2018. Disponível em: <http://nascecme.com.br/2014/wpcontent/uploads/2018/08/NANDA-I-2018_2020.pdf $>$. Acesso em: 10 Abr. 2020 .

8. Manganelli, Rigielli Ribeiro; Kirchhof, Raquel Soares; PieszakI, Greice Machado; Dornelles, Carla da Silveira. Intervenções de enfermeiros na prevenção de lesão por pressão em uma unidade de terapia intensiva. Rev. enferm. UFSM; 2019 jul. 15 [acesso em 2020 mar 03] Disponível em: https://periodicos.ufsm.br/reufsm/article/view/33881/pdf

9. Snaches, Bruna et al. Adesão da enfermagem ao protocolo de lesão por pressão em unidade de terapia intensiva. Arquivos de Ciências da Saúde, [S.1.], v. 25, n. 3, p. 27-31, dez. 2018. ISSN 2318-3691. Disponível em: $<$ http:// www.cienciasdasaude.famerp.br/index.php/racs/article/view/1058>. Acesso em: 29 abr. 2020. doi: https://doi.org/10.17696/2318-3691.25.3.2018.1058.

10. Vasconcelos, Josilene de Melo Buriti; Caliri, Maria Helena Larcher. Ações de enfermagem antes e após um protocolo de prevenção de lesões por pressão em terapia intensiva. Esc. Anna Nery, Rio de Janeiro , v. 21, n. 1, e20170001, 2017. Available from $<$ http://www.scielo.br/scielo.php?script=sci_arttext\&pid=S1414$81452017000100201 \& \operatorname{lng}=$ en\&nrm=iso $>$. access on 29 Apr. 2020. Epub Jan 16, 2017. http://dx.doi.org/10.5935/1414-8145.20170001.

11. Caldini, Luana Nunes; Araújo, Thiago Moura de; Frota, Natasha Marques; Barros, Lívia Moreira; Silva, Leonardo Alexandrino da; Caetano, Joselany Áfio. Avaliação de tecnologia educativa sobre lesão por pressão baseada em indicadores de qualidade assistenciais. Rev Rene (Online) ; 19: e32695, jan. - dez. 2018. [acesso em 29 abr 2020] Disponível em: http:// periodicos.ufc.br/rene/article/view/32695/pdf_1

12. Vangilder, C. et al. The International Pressure Ulcer Prevalence ${ }^{\mathrm{TM}}$ Survey. Journal Of Wound, Ostomy And Continence Nursing, [s.1.], 2017.

13. Zimmermann, Guilherme dos Santos et al. Predição de risco de lesão por pressão em pacientes de unidade de terapia intensiva: revisão integrativa. Texto contexto - enferm., , v. 27, n. 3, e3250017, 2018. Disponível em $<$ http://www.revenf.bvs.br/scielo.php?script=sci arttext\&pid=S0104$07072018000300500 \& \operatorname{lng}=$ pt\&nrm $=$ iso $>$. acessos em 29 abr. 2020. Epub 27-Ago-2018. http://dx.doi.org/10.1590/0104-07072018003250017.

14. Vendruscolo, Carine et al. Educação Permanente em Saúde de Santa Catarina: entre cenários e práticas. - 1.ed. - Porto Alegre: UNIDA, 2018. 331 p. : il. - (Série Atenção Básica e Educação na Saúde, v.17) Ebook ISBN: 
978-8554329-06-8 DOI: 10.18310/9788554329068 Disponivel em: < http:// historico.redeunida.org.br/editora/biblioteca-digital/serie-atencao-basicae-educacao-na-saude/educacao-permanente-em-saude-de-santa-catarinaentre-cenarios-e-praticas-pdf $>$ Acesso em: 16 mar. 2020.

15. Mazzo, Alessandra et al. Ensino de prevenção e tratamento de lesão por pressão utilizando simulação. Disponível em: https:/www.scielo.br/pdf/ ean/v22n1/pt_1414-8145-ean-2177-9465-EAN-2017-0182.pdf. Acesso em: 17 abr. 2020.

16. Silva, Elisama Gomes Correia et al . O conhecimento do enfermeiro sobre a Sistematização da Assistência de Enfermagem: da teoria à prática. Rev. esc. enferm. USP, São Paulo, v. 45, n. 6, p. 1380-1386, dez. 2011 . Disponível em $<\mathrm{http}$ //www.scielo.br/scielo.php?script=sci arttext\&pid=S0080-62342011000600015\&lng=pt\&nrm=iso $>$. acessos em 29 mar. 2020. https://doi.org/10.1590/S0080-62342011000600015.

17. Coren-ES. Codigo de Ética. Resolução Cofen $n^{\circ}$ 564/2017. Espirito Santo, 2017. Disponivel em: <http://www.coren-es.org.br/codigo-de-etica> Acesso em: 18 abr. 2020.

18. Silva, Camila Polo Camargo da; Dell'Acqua, Magda Cristina Queiroz; Corrente, José Eduardo; Castro, Meire Cristina Novelli e; Zornoff, Denise de Cássia Moreira. Construção do Aplicativo para o indicador de úlcera por pressão. J. health inform; 8(4): [134-141], out.-dez. 2016. ilus, graf. [acesso em 2020 abr 05] Disponível em: <http://www.jhi-sbis.saude.ws/ojs-jhi/ index.php/jhi-sbis/article/view/423/276>

19. Rolim, J. A. et al. Prevenção e tratamento de úlceras por pressão no cotidiano de enfermeiros intensivistas. Rev Rene. Fortaleza, v. 14, n. 1, p. $148-57,2013$.

20. Baron, Miriam Viviane et al. Estudo experimental com equipes de Enfermagem acerca do conhecimento sobre úlceras por pressão. Rev. Latino-Am. Enfermagem, Ribeirão Preto, v. 24, e 2831, 2016. Disponível em $\quad<$ http://www.scielo.br/scielo.php?script=sci arttext\&pid=S0104$11692016000100429 \& \operatorname{lng}=$ pt\&nrm=iso $>$. acessos em $\overline{2} 9$ abr. 2020. Epub 21-Nov-2016. http://dx.doi.org/10.1590/1518-8345.1134.2831.

21. Baquehai, Adna Ribeiro; Dallarosa, Fábia Sostisso. Conhecimento dos enfermeiros acerca da prevenção de lesões por pressão em unidade de terapia intensiva. Rev. enferm. UFPI ; 5(4): 13-18, out, -dez.2016. Disponível em: $<$ https://revistas.ufpi.br/index.php/reufpi/article/view/5426/pdf $>$ [acesso em: 15 Mar 2020] https://doi.org/10.26694/reufpi.v5i4.5426.

22. Lemos, Débora de Sousa et al. Conhecimentos dos profissionais de enfermagem relacionados às úlceras por pressão no centro de terapia semiintensiva. Revista de Enfermagem da UFSM, [S.1.], v. 4, n. 4, p. 751 - 760, jan. 2015. ISSN 2179-7692. Disponível em: <https://periodicos.ufsm.br/ reufsm/article/view/11707>. Acesso em: 29 abr. 2020. doi:http://dx.doi. org/10.5902/2179769211707.

23. Oliveira Carvalho, Francisco Matheus et al. The Nurses' Knowledge With Regards to Both Classification and Prevention of Pressure Injury / Conhecimento dos Enfermeiros sobre Classificação e Prevenção de Lesão por Pressão. Revista de Pesquisa: Cuidado é Fundamental Online, [S.1.], v. 11 , n. 3, p. 560-566, feb. 2020. ISSN 2175-5361. Disponível em: $<$ http:// www.seer.unirio.br/index.php/cuidadofundamental/article/view/6576>. Acesso em: 04 may 2020. doi:http://dx.doi.org/10.9789/2175-5361.rpcfo. v11.6576

24. Oliveira, Andrea Carvalho de; Garcia, Paulo Carlos; Nogueira, Lilia de Souza. Carga de trabalho de enfermagem e ocorrência de eventos adversos na terapiaintensiva: revisão sistemática. Rev. esc. enferm. USP, São Paulo, v. 50, n. 4, p. 683-694, ago. 2016. Disponível em $\quad<$ http://www.scielo.br/scielo.php?script=sci arttext\&pid=S008062342016000400683\&lng=pt\&nrm=iso $>$. acessos em $\overline{2} 9$ abr. 2020. http:// dx.doi.org/10.1590/S0080-623420160000500020.

25. Tonole, R. Elaboração e validação de instrumento para avaliação dos recursos humanos e materiais na perspectiva da prevenção de lesão por pressão: estudo metodológico. 93f. Dissertação (Mestrado Profissional em Enfermagem Assistencial). Escola de Enfermagem Aurora de Afonso Costa. Universidade Federal Fluminense. Niterói, 2019.

26. OPAS - Organização Pan-Americana da Saúde. Folha informativa - Envelhecimento e saúde. Disponivel em: < https://www.paho.org/bra/ index.php?option $=$ com_content $\&$ view $=$ article $\& i d=5661$ :follha-informativaenvelhecimento-e-saude $\&$ Itemid= $820>$ acesso em: 30 abr. 2020 .

27. Toffoletto, Maria Cecilia et al. Fatores relacionados à ocorrência de eventos adversos em pacientes idosos críticos. Rev. Bras. Enferm.,
Brasília , v. 69, n. 6, p. 1039-1045, dez. 2016. Disponível em $<$ http://www.scielo.br/scielo.php? script $=$ sci_arttext\&pid=S0034$71672016000601039 \& \operatorname{lng}=$ pt\&nrm $=$ iso $>$. acessos em 29 abr. 2020. http:// dx.doi.org/10.1590/0034-7167-2016-0199. 